\title{
The Effects of Resistance Training on Lower and Upper Body Strength Gains in Young Women
}

\author{
Paulo Gentil \\ College of Physical Education, University of Brasília, Brasília, DF, Brazil \\ Campus Universitário Darcy Ribeiro, Asa Norte, Brasília 70910970, DF, Brazil \\ E-mail: paulogentil@hotmail.com \\ João B. Ferreira-Junior (Corresponding author) \\ Federal Institute of Sudeste of Minas Gerais, Rio Pomba \\ José Sebastião da Paixão s/nº, Lindo Vale, Rio Pomba 36180000, MG, Brazil \\ E-mail: jbfjunior@gmail.com \\ Michael G. Bemben \\ Department of Health and Exercise Science, University of Oklahoma \\ Rm 115 HHC, Norman, OK 73019, USA. \\ E-mail: mgbemben@ou.edu \\ Diogo V. Ferreira \\ College of Physical Education, University of Brasília, Brasília, DF, Brazil \\ Campus Universitário Darcy Ribeiro, Asa Norte, Brasília 70910970, DF, Brazil \\ E-mail: ferreira.diogov@gmail.com \\ Martim Bottaro \\ College of Physical Education, University of Brasília, Brasília, DF, Brazil \\ Campus Universitário Darcy Ribeiro, Asa Norte, Brasília 70910970, DF, Brazil \\ E-mail: martim.bottaro@gmail.com
}

Received: 29-05- 2015

doi:10.7575/aiac.ijkss.v.3n.3p.18
Accepted: 22-07- 2015

Published: 31-07- 2015

URL: http://dx.doi.org/10.7575/aiac.ijkss.v.3n.3p.18

\begin{abstract}
It has been reported that hypertrophy gains is greater in upper body compared to lower body, however, there is no consensus that muscular strength gains are greater in upper body than in lower body. Therefore, the aim of the present study was to compare the strength gains between knee extensors and elbow flexors in response to similar resistance training regimens. Fifty five untrained young women (age: $21.6 \pm 2.9$ years, weight: $58.3 \pm 9.0 \mathrm{~kg}$ and height: $163.6 \pm$ $7.5 \mathrm{~cm}(\mathrm{Mean} \pm \mathrm{SD})$ ) participated in the study as volunteers. Resistance training was performed twice a week for 10 weeks. All subjects performed three sets of 8-12 maximum repetitions for leg press, knee flexion, lat pull down and bench press exercises. Unilateral knee extensors and elbow flexors peak torque (PT) were measured before and after the training period by performing two sets of four repetitions at $60^{\circ} \mathrm{s}^{-1}$, on an isokinetic dynamometer. There were significant increases in PT for both elbow flexors (11.74\% [8.0, 17.7], $\mathrm{p}<0.05)$ and knee extensors $(11.45 \%$ [9.2, 15.3], $\mathrm{p}<0.05)$ with no differences between muscle groups $\mathrm{p}>0.05)$. However, there was no correlation between gains in knee extensors and elbow flexors PT. The analysis of knee extensors PT lead to the formation of two clusters groups: 1) High responders $(n=10): 28.29 \pm 8.74 \%$ and 2) Low-responders $(n=37): 7.94 \pm 5.95 \%$. Both groups had significant increases in knee extensors PT, however, increases in the high responders were higher than in low responders $(p<0.05)$. These results suggest that upper- and lower body muscles present similar strength gains after similar resistance training regimens in untrained young women, although individual muscle response may vary in upper and lower body muscles.
\end{abstract}

Keywords: Knee extensors, Elbow flexors, Peak torque

\section{Introduction}

Resistance training is widely used in order to induce gains in muscle strength and hypertrophy (ACSM, 2009; Bottaro, Veloso, Wagner, \& Gentil, 2011; Kraemer et al., 2002), however, some muscle groups seems to be less responsive, while others seem to be more reactive (Wernbom, Augustsson, \& Thomee, 2007). In relation to muscle hypertrophy it has been reported that cross sectional area of the elbow flexors tended to increase at a greater extent than the quadriceps muscle (Abe, DeHoyos, Pollock, \& Garzarella, 2000; Cureton, Collins, Hill, \& McElhannon, 1988; Housh, Housh, 
Johnson, \& Chu, 1992a; Welle, Totterman, \& Thornton, 1996). In this sense, Wernborn et al. (Wernbom et al., 2007) reported that muscle cross sectional area of the elbow flexors could increase at a rate of $0.20 \%$ per day, while quadriceps muscle increases at a rate of $0.11 \%$.

However, there is no consensus about the hypothesis that muscular strength gains are greater in upper body when compared to lower body (Abe et al., 2000; Cureton et al., 1988; Housh et al., 1992a; Lexell, Downham, Larsson, Bruhn, \& Morsing, 1995; Welle et al., 1996). Housh et al. (Housh et al., 1992a) found that strength gains in arm muscles were higher than leg muscles in response to isokinetic strength training. Abe et al. (Abe et al., 2000) has reported that resistance training produces a higher strength increase in the upper body compared to the lower body for women, but not for men. In contrast, Welle et al. (Welle et al., 1996) and Lexell et al. (Lexell et al., 1995) found greater strength gains in the lower body when compared to the upper body. These controversial results may be due to differences in strength training protocols applied for knee extensors and elbow flexors in these studies, specifically in the number of exercises and sets performed for different muscles (Abe et al., 2000; Cureton et al., 1988; Housh et al., 1992a; Lexell et al., 1995; Welle et al., 1996).

Assessments that involve upper body strength are important predictors of mortality (Cooper, Strand, Hardy, Patel, \& Kuh, 2014; Kim et al., 2015; Ruiz et al., 2008). Therefore, assessing the specific responses of upper and lower body muscles to training is important to correctly define the training volume necessary to induce the desired adaptations. This is particularly important in women, as they are commonly believed to have limited upper body strength and culturally perform less activities that involve the muscles of this region. This brings the necessity to perform specific studies in women, as previous studies involved men (Housh, Housh, Johnson, \& Chu, 1992b) or had mixed sample with a low number of women (Cureton et al., 1988; Welle et al., 1996).

The investigation of the responses of upper and lower body muscles of women to similar resistance training programs would provide important information for designing resistance training programs, helping to comprehend, avoid and/or correct muscle imbalances between different body muscles. Therefore, the purpose of this study was to compare strength gains between knee extensors and elbow flexors to similar resistance training regimens in untrained young women. Our hypothesis was that strength gains would be similar in knee extensors and elbow flexors muscle groups.

\section{Materials and Methods}

\subsection{Subjects}

Fifty five young women (age: $21.6 \pm 2.9 \mathrm{yrs}$, weight: $58.3 \pm 9.0 \mathrm{~kg}$ and height: $163.6 \pm 7.5 \mathrm{~cm}(\mathrm{Mean} \pm \mathrm{SD})$ ) volunteered for the study. The volunteers were recruited through folders and advertising banners around the University Campus. To be accepted, participants had to be at least 18 years old, had not been participating in any resistance training program over the past six months and were free of health problems, which was attested by a medical exam. Only the participants that attended at least $80 \%$ of the training sessions were included in the final analysis (Gentil \& Bottaro, 2013). The volunteers were instructed not to change their nutritional habits and, if any relevant change were detected (e.g. becoming a vegetarian, being on caloric restriction, use of nutritional supplements or ergogenics substances, etc.) the data of the participants were excluded from the analyses. Data of volunteers were excluded for failing to meet the inclusion criteria: low attendance $(n=5)$ and performance of additional resistance training $(n=3)$. The characteristics of the excluded participants did not differ from the others. All volunteers were notified about the experimental procedures, benefits and risks before signing the informed consent form. An Institutional Research Ethics Committee granted approval for the study.

\subsection{Experimental design}

After familiarization, all subjects performed 10 weeks of a strength training program, in a total of 20 training sessions divided into two nonconsecutive training days per week. Strength measurements in the knee extensors and elbow flexors were taken on an isokinetic dynamometer before and after the training period. Differences between knee extensors and elbow flexors strength gains in response to resistance training regimens were compared.

\subsection{Resistance training protocol}

Resistance training was performed twice a week for 10 weeks based on the American College of Sports and Medicine recommendations (ACSM, 2009). All subjects performed the same exercises, following the same order (leg press, knee flexion, lat pull down and bench press - GervaSport, Cotia, Brazil). Elbow flexion exercise was not included in the resistance exercise training program since previous data from our lab has determined that adding elbow flexion exercise to a program that already includes lat pull down exercise has no added benefit regarding strength gains and hypertrophy gains for the elbow flexors compared to only performing lat pull down exercises (Gentil et al., 2013). All exercises were performed with three sets of 8-12 maximum repetitions (RM). Subjects were instructed to perform the concentric and eccentric phases each in two seconds, without pause between contractions. During the training sessions, music tracks with $120 \mathrm{bpm}$ were played in order to facilitate control of movement velocity (Gentil et al., 2013). Participants were oriented to perform all sets until concentric failure. If necessary, loads were adjusted from set to set to maintain the designated number of repetitions. Training sessions were closely supervised by experienced trainers, because previous research has demonstrated greater gains in supervised vs. unsupervised training (Gentil \& Bottaro, 2010). 
Training was conducted two days a week, with a minimum of 48 hours between sessions. Rest intervals between sets ranged from 1.5 to 2.5 minutes. All training logs were verified by a supervisor following every exercise session.

\subsection{Peak Torque (PT) assessment}

Unilateral knee extension and elbow flexion peak torque (PT) were tested using two sets of four repetitions at $60^{\circ} \mathrm{s}^{-1}$, on a Biodex System 3 isokinetic dynamometer (Biodex Medical, Inc., Shirley, NY) with 60s rest between sets. Calibration of the dynamometer was performed according to the manufacturer's specifications before every testing session. Knee extensors assessment procedures were as follows: the participants sat upright with the axis of rotation of the dynamometer arm oriented with the lateral femoral condyle of the right knee. Belts were used to secure the thigh, pelvis, and trunk to the dynamometer chair to prevent additional body movement. The chair and dynamometer settings were recorded to ensure the same positioning for all tests. The flexor torque produced by the relaxed segment was used for gravity correction. Participants were instructed to fully extend and flex the knee and to work maximally during the test. Elbow flexors assessment was as follows: the participants were seated on an arm curl bench with the exercised upper arm supported at $45^{\circ}$ of shoulder abduction and their elbow aligned with the axis of rotation of the dynamometer's lever arm. The forearm remained in a supinated position throughout the test session. Verbal encouragement was given throughout the test. The procedures were administered by the same investigator (Brown, 2000). Baseline test and retest intraclass correlation coefficient (ICC) and standard error of the mean (SEM) for peak torque were 0.98 and $2.3 \%$ for knee extensors, and 0.96 and $2.4 \%$ for elbow flexors.

\subsection{Statistical analysis}

Normal distribution of the data was confirmed using the Kolmogorov-Smirnov tests. All values were reported as means and standard deviations. PT alterations were calculated using the formula (Post-Pre)/Pre*100\%. The interaction between PT and time was evaluated by a two-way repeated measures ANOVA with a $2 \times 2$ design [muscle group (Elbow flexor and Knee extensor) $\mathrm{x}$ time (pre and post training)]. Multiple comparisons with confidence interval adjustment by the LSD method were use for post hoc comparisons when necessary.

Pearson linear correlation coefficients were calculated to analyze the association between mean change in PT of the elbow flexors and knee extensors. Two-step cluster analysis was used to create homogenous groups according to elbow flexors and knee extensors PT alterations and Pearson chi-square was used to analyze association between the distribution of elbow flexors and knee extensors PT alterations. Significance level was set at $\mathrm{P}<0.05$.

\section{Results}

There was a significant interaction for PT and time $(\mathrm{p}<0.05$, power= 1.0). There were significant increases in PT for both elbow flexors and knee extensors after 10 weeks of resistance training (Table 1). However, there was no significant difference in mean change in PT between knee extensors and elbow flexors $(\mathrm{p}>0.05$, power $=0.058)$.

Table 1. Elbow flexors (EF) and knee extensors (KE) peak torque strength before and after 10 weeks of resistance training.

\begin{tabular}{lccc}
\hline & Pre-training & Post-training & $\Delta$ (post - pre training) \\
\hline EF peak torque (N.m) & $25.1 \pm 4.9$ & $28.0 \pm 5.5^{*}$ & $11.74 \%$ \\
KE peak torque (N.m) & $138.1 \pm 26.9^{\#}$ & $154.0 \pm 26.9^{* \#}$ & $11.45 \%$
\end{tabular}

$(*) \mathrm{p}<0.05$, higher than pre-training. (\#) $\mathrm{p}<0.05$, higher than EF.

Two clusters groups were formed for elbow flexors PT alterations: 1) Responders ( $\mathrm{n}=18): 30.65 \pm 11.6 \%$ and 2) Nonresponders $(\mathrm{n}=29): 1.83 \pm 8.12 \%$. The increases in elbow flexors PT were significant for responders $(\mathrm{p}<0.05)$ but nor for non-responders ( $\mathrm{p}>0.05$ ). The analysis of knee extensors PT lead to the formation of two clusters groups: 1) High responders ( $\mathrm{n}=10): 28.29 \pm 8.74 \%$, and 2) Low-responders $(\mathrm{n}=37): 7.94 \pm 5.95 \%$. Both groups had significant increases in knee extensors PT, however, increases in the high responders were higher than in low responders $(\mathrm{p}<0.05)$. Figure 1 shows individual response for both elbow flexors and knee extensors PT alterations. According to the results, there was no significant association between the distribution of elbow flexors and knee extensors PT clusters ( $>>0.05)$. Also, there was not a significant correlation between change in PT of the knee extensors and elbow flexors $(r=0.14, p>0.05)$. 


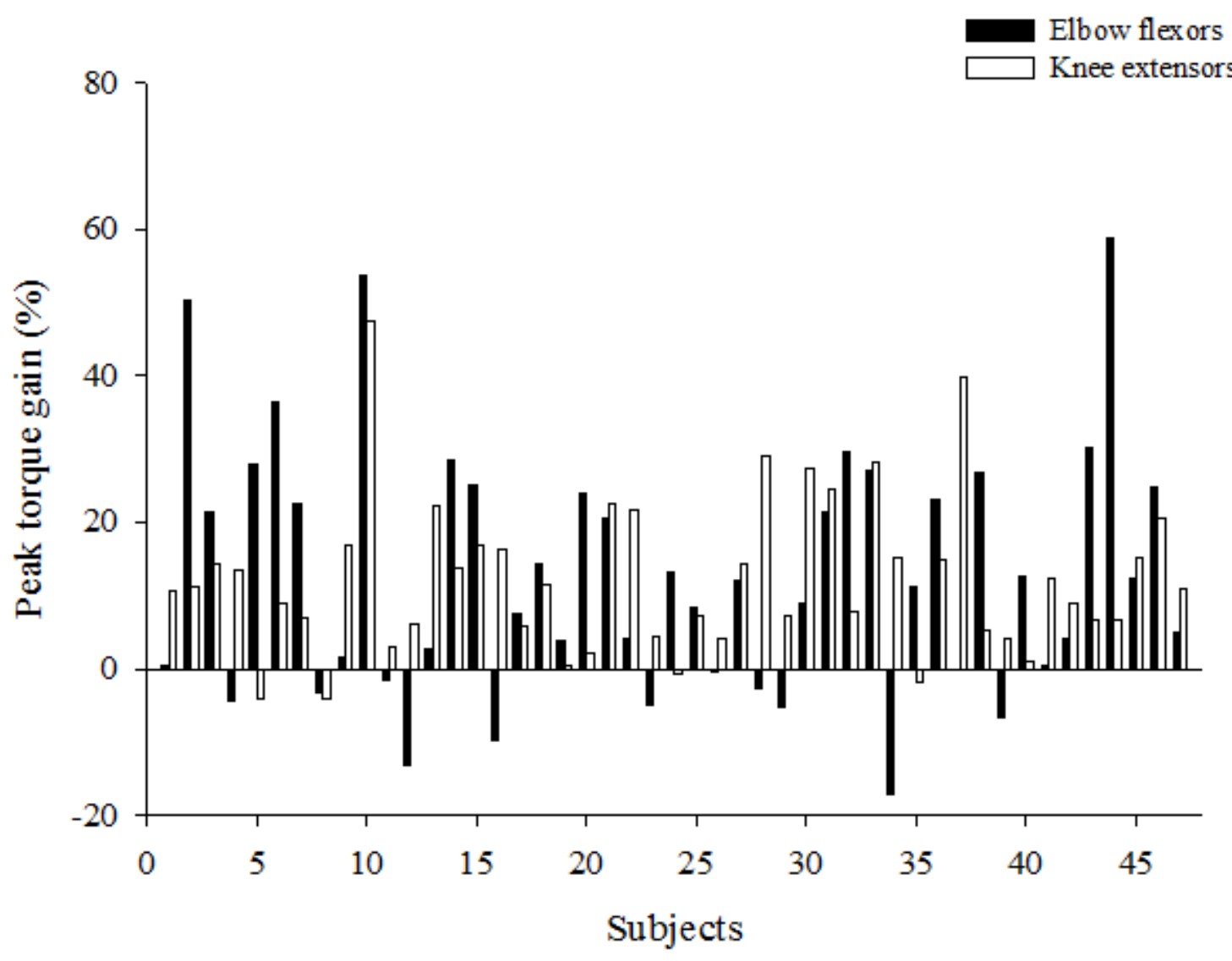

Figure 1. Individual peak torque gains in elbow flexors and knee extensors after 10 weeks of resistance training.

\section{Discussion}

The aim of the present study was to compare strength gains between knee extensors and elbow flexors in response to similar resistance training regimens. The main finding of the present study was the similar mean strength gains observed in the elbow flexors and knee extensors after 10 weeks of resistance training. Thus, this result supports the hypothesis that, in general, strength gains in knee extensors and elbow flexors induced by similar resistance training programs would be similar in young women. It is important to emphasize that the present study is the first to directly compare upper and lower body strength gains in women. Moreover, the main difference among our study and most of those which reported greater strength gains in upper body (Abe et al., 2000; Cureton et al., 1988; Housh et al., 1992a) or lower body muscles (Lexell et al., 1995; Welle et al., 1996), seems to be the differences in training volume for different muscle groups and the characteristics of the participants.

It has been previously reported that resistance training may induce greater strength gains in the lower body when compared to the upper body (Lexell et al., 1995; Welle et al., 1996). Welle et al. (Welle et al., 1996) found higher gains in knee extensors strength $(38 \%)$ in comparison to elbow flexors $(21 \%)$ in young men after 3 months of resistance training, even though the training volume for elbow flexors was higher than knee extensors, since there was two exercises involving the elbow flexors and only one for the knee extensors. When using similar resistance training regimens for upper and lower body muscles, Lexell et al. (Lexell et al., 1995) observed an increase of $49 \%$ for elbow flexion 1RM and $163 \%$ for knee extension in elderly subjects in response to 11 weeks of training.

On the other hand, Cureton et al. (Cureton et al., 1988) used similar training regimens for the upper and lower body muscles and observed strength gains ranging from $36.2 \%$ to $59.2 \%$ in the elbow flexors and 12.8 to $24.4 \%$ for the knee extensors, after 16 week training in young men and women. Similarly, Housh et al. (Housh et al., 1992a) found higher strength increases in upper muscles in comparison with the lower body in untrained male college students. The subjects performed 6 sets of 10 repetitions of extension and flexion of the non-dominant leg and arm at $120^{\circ} . \mathrm{s}^{-1}$ during 8 weeks and found a significant strength increase of $20.6 \%$ for elbow flexors $(p<0.05)$, while the $11.2 \%$ increase in the knee extensors strength was not significant. In the same way, Abe et al. (Abe et al., 2000) reported greater strength gains in the upper body (27\%) compared to the lower body muscles (19\%) for women after 12 weeks of weight training, but it is important to note that volunteers performed a greater training volume for elbow flexors when compared to knee extensors.

Although the mean strength gains between knee extensors and elbow flexors were similar in the present study, there was not a significant correlation between them and there was no statistically significant association between knee extensors and elbow flexors strength responsiveness. Therefore, one possible explanation to the divergence in the literature is that some studies involved persons more prone to gain strength in specific muscles groups. Moreover, the 
lack of correlation between gains in specific body parts could have important implications for talent detection and training, since a favorable phenotype for strength in a muscle or muscle group does not necessarily imply that this is valid for other muscle groups.

Another possible explanation is specificity in gender response. Contrary to the present study, the studies with mixed samples (Cureton et al., 1988; Welle et al., 1996) and involving only males (Housh et al., 1992b) reported greater strength gains in the upper body compared to lower body muscles. This can suggest a specific gender response, as the higher distribution of muscle mass in the upper body found in men can induce an altered response to training in comparison to women.

Still, our results are different from those reported by Abe et al. (Abe et al., 2000), who analyzed young women. According to the results, 1RM increased by $37.5 \%$ in the chest press and $23.4 \%$ in the knee extension in women, suggesting a higher response in upper body muscles. However, the training protocol involved only one exercise for the knee extensors (knee extension), and two for the muscles that act in the chest press (elbow extension and chest press). Moreover, the comparison of a multi joint exercise with a single joint may have interfered with the results as learning may have cause the load to increase more in the chest press.

Considering that muscle strength gain induced by resistance training depends on both neural and morphological adaptations (ACSM, 2009; Kraemer et al., 2002), and that muscle hypertrophy is greater in upper than lower body muscles (Abe et al., 2000; Cureton et al., 1988; Housh et al., 1992a; Welle et al., 1996), one could suggest that similar strength increases between elbow flexors and knee extensors in response to resistance training could be due to greater neural adaptations in knee extensors. However, since the present study did not assess neural adaptations and muscle hypertrophy, this hypothesis remains speculative and needs to be further investigated.

\subsection{Practical Applications}

To promote chronic gains in muscle strength, athletic trainers and conditioning professional should include preplanned variations and systematic periodization within an overall strength training program. One important variable in designing resistance training is the balance of training between muscle groups in order to avoid an/or correct imbalances between them (i.e. upper and lower body or agonist and antagonist). Our results showed that, in general, there is no difference in upper and lower body strength responsiveness and that training programs should be individually designed taking into account the non-uniform muscle strength responsiveness to training in untrained women.

\section{Conclusions}

In conclusion, mean strength increase seems to be similar for elbow flexors and knee extensors in response to resistance training in women, but it is important to note that great individual differences exist. More studies on this topic are necessary in order to provide grounds for a more precise conclusion and understanding the responsiveness of muscle groups to resistance training. In addition, it is important evaluate other populations, such as athletes and young men and elderly.

\section{Acknowledgements}

The study was partially funded by Coordination for the Improvement of Higher Level -or Education- Personnel (CAPES) - Brazil, and National Counsel of Technological and Scientific Development (CNPq) - Brazil.

\section{References}

Abe, T., DeHoyos, D. V., Pollock, M. L., \& Garzarella, L. (2000). Time course for strength and muscle thickness changes following upper and lower body resistance training in men and women. European Journal of Applied Physiology, 81(3), 174-180.

American College of Sports Medicine position stand. Progression models in resistance training for healthy adults. (2009). Medicine Science in Sports and Exercise, 41(3), 687-708.

Bottaro, M., Veloso, J., Wagner, D., \& Gentil, P. (2011). Resistance training for strength and muscle thickness: Effect of number of sets and muscle group trained. Science \& Sports, 26(5), 259-264.

Brown, L. E. (2000). Isokinetics in human performance. Champaign, Ill.: Human Kinetics.

Cooper, R., Strand, B. H., Hardy, R., Patel, K. V., \& Kuh, D. (2014). Physical capability in mid-life and survival over 13 years of follow-up: British birth cohort study. British Medical Journal, 348, g2219.

Cureton, K. J., Collins, M. A., Hill, D. W., \& McElhannon, F. M., Jr. (1988). Muscle hypertrophy in men and women. Medicine Science in Sports and Exercise, 20(4), 338-344.

Gentil, P., \& Bottaro, M. (2010). Influence of supervision ratio on muscle adaptations to resistance training in nontrained subjects. The Journal of Strength and Conditioning Research, 24(3), 639-643.

Gentil, P., \& Bottaro, M. (2013). Effects of training attendance on muscle strength of young men after 11 weeks of resistance training. Asian Journal of Sports Medicine, 4(2), 101-106. 
Gentil, P., Soares, S. R., Pereira, M. C., Cunha, R. R., Martorelli, S. S., Martorelli, A. S., \& Bottaro, M. (2013). Effect of adding single-joint exercises to a multi-joint exercise resistance-training program on strength and hypertrophy in untrained subjects. Applied Physiology, Nutrition, and Metabolism, 38(3), 341-344.

Housh, D. J., Housh, T. J., Johnson, G. O., \& Chu, W. K. (1992). Hypertrophic response to unilateral concentric isokinetic resistance training. Journal of Applied Physiology (1985), 73(1), 65-70.

Kim, Y. H., Kim, K. I., Paik, N. J., Kim, K. W., Jang, H. C., \& Lim, J. Y. (2015). Muscle strength: A better index of low physical performance than muscle mass in older adults. Geriatrics \& Gerontology International. Epub ahead of print.

Kraemer, W. J., Adams, K., Cafarelli, E., Dudley, G. A., Dooly, C., Feigenbaum, M. S., Triplett-McBride, T. (2002). American College of Sports Medicine position stand. Progression models in resistance training for healthy adults. Medicine Science in Sports and Exercise, 34(2), 364-380.

Lexell, J., Downham, D. Y., Larsson, Y., Bruhn, E., \& Morsing, B. (1995). Heavy-resistance training in older Scandinavian men and women: short- and long-term effects on arm and leg muscles. Scandinavian Journal of Medicine and Science in Sports, 5(6), 329-341.

Ruiz, J. R., Sui, X., Lobelo, F., Morrow, J. R., Jr., Jackson, A. W., Sjostrom, M., \& Blair, S. N. (2008). Association between muscular strength and mortality in men: prospective cohort study. British Medical Journal, 337, a439.

Welle, S., Totterman, S., \& Thornton, C. (1996). Effect of age on muscle hypertrophy induced by resistance training. The journals of gerontology. Series A, Biological sciences and medical sciences, 51(6), M270-275.

Wernbom, M., Augustsson, J., \& Thomee, R. (2007). The influence of frequency, intensity, volume and mode of strength training on whole muscle cross-sectional area in humans. Sports Medicine, 37(3), 225-264. 